\title{
Volume Estimation of Various Brain Components Using MR Images - A Technical Report
}

\author{
Fuhua Chen* and Katherine Hastings
}

West Liberty University, West Virginia, USA

\begin{abstract}
In this paper, we present and discuss issues related to volume estimation of various brain components using MR brain images. We discuss pre-processing techniques to remove elements such as skull, blood vessels and fats from the MR images since these are non-essential to the volume calculations. The volume estimation is based on image segmentation. A challenge in MR brain image segmentation is to distinguish central gray matter from surrounding matters. This paper provides a frame work from pre- processing stage through volume estimation for white matter, gray matter and cerebrospinal fluid (CSF). The main contribution of this paper contains two parts. First, it provides a software-based method for interactive image pre-processing; second, it introduces a software-based, supervised interactive image segmentation method to deal with the segmentation of different matters, especially the central gray matter. Experiments with real data demonstrate the efficiency of our frame work.
\end{abstract}

Keywords: Image segmentation; Magnetic Resonance (MR) images; Central gray matter

\section{Introduction}

Traumatic brain injury happens when brain is hurt by an external force. Many imaging techniques, such as Computed Tomography (CT), Magnetic Resonance imaging (MR), and Positron Emission Tomography (PET), have been used in the diagnosis and treatment of such injuries. In the project "Biochemical Markers of Traumatic Brain Injury", we attempted a pathological analysis of diseases related to the brain by using MR images to estimate the change in volumes of different brain tissues. This paper is a technical report of part of the project focusing on volume estimations of white matter, gray matter, and Cerebrospinal Fluid (CSF). Volume estimation of different matters is especially important in this project. The key step in volume estimation based on images for the brain matters is image segmentation [1]. However, there are two main challenges that arise during this process.

The first challenge is how to pre-process the raw data so that it can be easily segmented and the volumes of different matters can be easily estimated. The raw MR data for a person's brain is a series of twodimensional DICOM data that contains the skull, cerebrum, cerebellum and other organ matters in addition to the white matter, gray matter and CSF of interest. This data needs to be converted to a format that is easier to process using softwares such as $\mathrm{C}++$ or MATLAB. In addition, it is necessary to remove the non-essential part pertaining to tissues and matter other than white matter, gray matter and CSF.

The second main challenge is the differentiation of various matters only based on intensities. The big trouble is of the central gray matter. Central gray matter is the gray matter located around the cerebral aqueduct within the tegmentum of the midbrain [2]. In most settings of MR imaging, white matter has the strongest intensity, gray matter a mild intensity, and cerebrospinal fluid the weakest intensity. However, the intensity of central gray matter in a MR brain image is often very similar to the intensity of white matter in the surface layer of the brain. As a result, it is difficult to approximately distinguish central gray matter from white matter. If the threshold for gray matter is set higher, then the white matter at surface layer may be misclassified as gray matter; if the threshold for gray matter is set lower, then some central gray matter may be misclassified as white matter. Many unsupervised segmentation methods have been developed to address this issue, such as non-local methods [3,4] and bias-correction methods [5-7]. However, researchers have not yet overcome such a dilemma efficiently due to the inherent closeness between the intensity of central gray matter and the intensity of white matter located at the surface layer of the cerebrum.

The intensities of blood and fat can also be very strong in MR brain images, which can lead to their misclassification as white matter. In order to estimate the volume for white matter more precisely, those high-intensity parts that are not white matter must be removed prior to unsupervised image segmentation. Lastly, the cerebellum must also be separated since only the functions of cerebrum is concerned in this project.

In this paper, we deal with the first challenge by integrating some existing soft- wares, and deal with the second challenge by developing a new software so that the resulted data after unsupervised segmentation can be refined using supervised and interactive way.

\section{Materials}

Most of the raw MR brain images used in this paper were provided by Shands Hospital in Gainesville, Florida, while some were obtained from various hospitals in Jacksonville, Florida. All the data has a DICOM format and is provided by either patients (as abnormal data) or volunteers (as normal data).

\section{Procedures}

The overall procedure can be broken into three stages. In the first stage, we pre-process the data so that the brain images contain only matters of interest which will be applied to an unsupervised

${ }^{*}$ Corresponding author: Fuhua Chen, West Liberty University, West Virginia USA, Tel: 3043365000; E-mail: fuhua.chen@westliberty.edu

Received December 24, 2014; Accepted February 27, 2015; Published March 10, 2015

Citation: Chen F, Hastings K (2015) Volume Estimation of Various Brain Components Using MR Images - A Technical Report. J Appl Computat Math 4: 207. doi:10.4172/2168-9679.1000207

Copyright: ( $) 2015$ Chen F, et al. This is an open-access article distributed under the terms of the Creative Commons Attribution License, which permits unrestricted use, distribution, and reproduction in any medium, provided the original author and source are credited. 
image segmentation algorithm; In the second stage, an unsupervised image segmentation is applied to the data, and then a supervised segmentation is used to adjust and refine the segmentation. Finally, the segmentations are converted to a volume calculation. The overall procedures can be outlined below:

\section{Overall steps:}

\section{Pre-processing}

2. Image segmentation and adjustment and/or refinement

\section{Volume estimation}

\section{Pre-processing}

The first step of pre-processing is to remove skull from original MR brain images. We used the software FSL. It is a comprehensive library of analysis tools for FMRI, MR and DTI brain imaging data. The software is developed by Oxford University and can be easily download and installed. It is free for non-profitable use. As the input data is required to be either Analyze-format or NIFTI-format, we first use MRIcro to convert DICOM data to either of Analyze-format or of NIFTI-format. The detailed steps are listed below.

\section{Steps for pre-processing}

1. Use MRIcro (Version: 1.40 build 1) to transform a series of 2D DICOM data into a 3D Analyze data (.img). The software was developed by Chris Rorden's Neuropsychology Lab and can be downloaded from their official website.

2. Use FSL (Version: 4.1.7) to remove the skull from the given 3D Analyze-format brain file (.img) or NIFTI-format brain file (.nii). The software FSL is developed by Oxford University and can be free download from their official website.

3. Use the developed software "BrainImg Processing" to further process the data. Several slices of the original DICOM data of a set of MR images from one brain are shown in Figure 1.

Figure 2 shows the data after the skull was removed in Step 2. Note there are still some parts that are not main matters of interest, such as blood and fats, which can drastically affect our volume estimations. The removing of these parts is carried out in Step 3 using the developed software. Figure 3 highlights these elements with the red circle

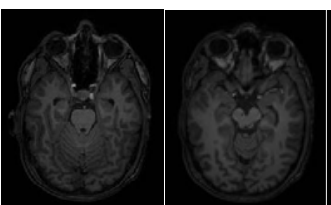

(a)

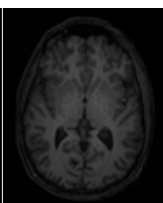

(c)

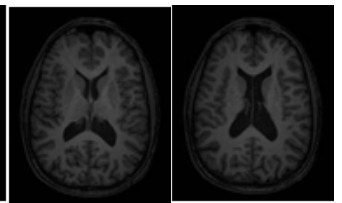

(d)

(e)
Figure 1: Original data.

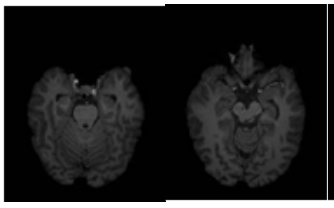

(a)

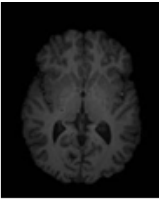

(c)

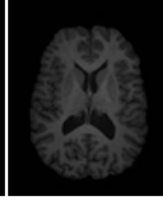

(d)

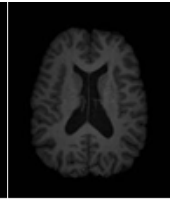

(e)
Figure 2: Brain images without skull.

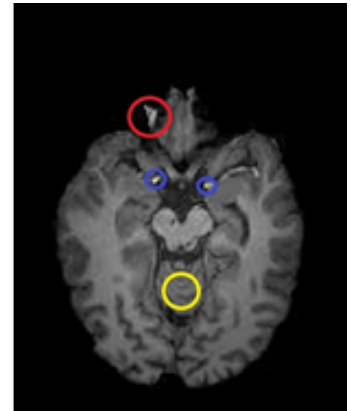

Figure 3: Brain images with non-essential matters identified.

identifying fat, the blue circle identifying blood, and the yellow circle identifying the cerebellum.

Although there have been papers addressing automatic cerebellum segmentation $[8,9]$, the removal is usually neither clear nor precise for real images. In our software BrainImg Processing, we provide an interactive way to remove blood and fats, as well as the cerebellum. Figure 2 shows the main images following the removal of blood, fat, and the cerebellum.

\section{Image segmentation}

After pre-processing the raw MR brain images, we apply image segmentation to the resulting images using a multiphase soft image segmentation method $[6,10]$. This process is embedded in the BrainImg Processing software. The image segmentation model is given below.

where I $(\mathrm{x})$ is the image to be segmented, ci and $\sigma \mathrm{i}$ are the mean and the deviation of the $\mathrm{i}$-th phase, $\mathrm{K}$ is the number of phases, $\mathrm{pi}(\mathrm{x})$ denotes the percentage of pixel $x$ belonging to the $\mathrm{i}$-th phase, and $\nabla \mathrm{pi}$ is the gradient of pi. In this application, $K=3$ because there is one phase for each brain matter of interest, namely, white matter, gray matter, and CSF. The first term in the equation is used to force the segmentation to fit the given image, and the second term (called total variation of pi ) is used to force smoothness for each phase (or, in this context, each type of brain matter) (Figure 4).

\section{Adjustment and refinement}

As we mentioned at the beginning of the paper, the intensity of central gray matter is usually close to the intensity of white matter in the surface layer of cerebrum. Therefore, the unsupervised segmentation may not be ideal. The central gray matters of images Figure $5 \mathrm{a}, \mathrm{b}$ and $\mathrm{c}$ are highlighted in Figure 4a, b and c, respectively.

After applying the unsupervised segmentation, most of the matters were classified correctly. However, due to the closeness of intensities between central gray matter and white matter, much central gray matter was misclassified to white matter. There have been some papers addressing the problem of inhomogeneity of intensities, such as biascorrection based multiphase image segmentations [5,7,11-13] and nonlocal information or global information based image segmentations $[3,4]$. However, all of those methods, including the unsupervised segmentation method employed inBrainImg Processing, do not work very well for real MR brain images due to the existence of central gray matter. Therefore, in BrainImg Processing, we added two functions, namely, adjustment and refinement which will be briefly described. These functions are especially useful for experienced doctors who can distinguish between white matter and central gray matter on MR brain images. 
1. The adjustment function provides a supervised image segmentation framework.

It assigns some regions to each matter based on prior knowledge and then uses the assigned matter as a reference to determine to which matter its neighborhood belongs.

2. The refinement function provides a way for local image segmentation. The reason that gray matter could not be segmented correctly using an unsupervised segmentation is that the intensity of central gray matter is much higher than the intensity of gray matter at the surface layer of the brain, and is close to (or even higher than) the intensity of white matter in the outer layer (not in the central region). The refinement function allows users to choose a central region and then apply a local image segmentation only for the chosen region. In this way, a more precise segmentation can be achieved.

The segmentation results of images in Figure $5 \mathrm{a}, \mathrm{b}$ and $\mathrm{c}$ using unsupervised segmentation are shown in the first row in Figure 6. From the images, we can see that much of the central gray matter is misclassified as white matter, while some white matter near the surface layer of the cerebrum are misclassified as gray matter. The results after refinement are shown in the second row in Figure 6.

\section{Introduction to BrainImg Processing}

BrainImg Processing is a software that was developed in our project to address two main issues. First, it can be used to manually remove some parts of an image. Second, when the image segmentation is not precise enough, it can be used to refine or adjust the segmentation.

Although there have been papers addressing automatic (or unsupervised) cerebellum segmentation [8,9] the removing is usually neither clear nor precise for real images. Moreover, when using FSL to remove skull from an given image, it is sometime either overremoved or under-removed. In addition, all fats and blood vessels must be removed from the images in our project. All of these tasks can be achieved with the software BrainImg Processing. For instance, if the skull is under-removed, it can be polished manually using BrainImg Processing by selecting unnecessary parts and then choosing "Removing". Fats and blood vessels can be similarly removed. When a proper set of parameters are chosen to apply an image segmentation and those parts near the surface layer of the cerebrum are segmented

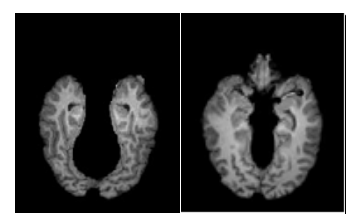

(a) (b)

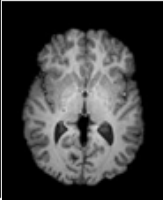

(c)

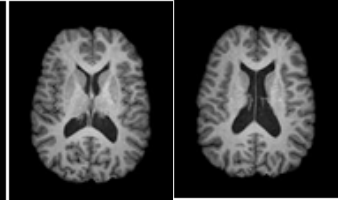

(d)

(e)
Figure 4: Matters of interest.

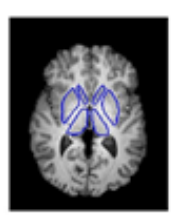

(a)

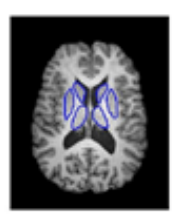

(b)

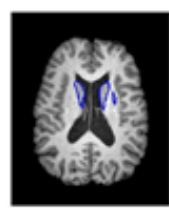

(c)
Figure 5: Central gray matter.
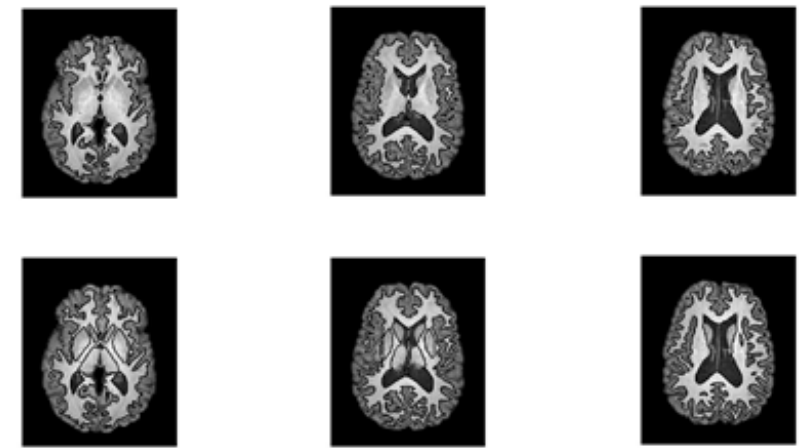

Figure 6: Comparison between refined and non-refined brain images

ideally, the central gray matter is usually under-segmented. That is, much of the central gray matter is misclassified as white matter. In this case, the software provides two options for the refinement or adjustment of the segmentation. One option is to choose a region that is not segmented correctly, and then apply the segmentation locally using adjusted parameters. The second option is to use the software to assign some regions to each matter, and then run supervised image segmentation. Both options can achieve a better result.

\section{Volume estimations}

After the image segmentation, three phases are obtained. Roughly speaking, the highest-intensity phase (denoted by P1) corresponds to white matter, the median-intensity phase (denoted by P2) corresponds to gray matter, and the lowest-intensity phase (denoted by P3) corresponds to cerebrospinal fluid. The P3 phase, however, contains more than just cerebrospinal fluid. In addition to the dark background of the image (whose intensity is similar to that of CSF), when fat and blood are removed from an image, the result is also a dark region. Therefore, the P3 phase is a combination of CSF, background, and "missing" fat and blood.

Suppose that the resolution of the $3 \mathrm{D}$ MR brain image is 1 millimeters $\times \mathrm{m}$ millimeters $\times \mathrm{h}$ millimeters (In our project, the resolution is $1 \mathrm{~mm} \times 1 \mathrm{~mm} \times 1 \mathrm{~mm}$ ). Then the algorithm for calculating the volumes for different matters is as below.

Steps for volume estimation for different matters:

1. Find the total number of voxels for the sum of all three matters, denoted by $\mathrm{N}$; this can be easily obtained by setting a threshold a little bigger than the intensity of the background.

2. Calculate the total number of voxels for Phase P1, denoted by N1;

3. Calculate the total number of voxels for Phase P2, denoted by N2;

4. The volume of white matter is obtained by $\mathrm{N} 1 \times 1 \mathrm{mh}$ millimeters;

5 . The volume of gray matter is obtained by $\mathrm{N} 2 \times 1 \mathrm{mh}$ millimeters;

6. The volume of cerebrospinal fluid is obtained by $(\mathrm{N}-\mathrm{N} 1 \mathrm{~N} 2) \times \mathrm{l}$ $\mathrm{mh}$ millimeters. The volumes for different matters can be shown automatically by the software after each segmentation or adjustment.

\section{Conclusion}

In this paper, we give a technical report on a previous project related to MR brain image processing. The first part of the paper discusses preprocessing of data using existing softwares. We then focus on issues 
Citation: Chen F, Hastings K (2015) Volume Estimation of Various Brain Components Using MR Images - A Technical Report. J Appl Computat Math 4: 207. doi:10.4172/2168-9679.1000207

Page 4 of 4

related to volume estimation of various brain matters in MR brain images. Namely, we discuss and analyze the problem of central gray matter segmentation. We point out that, based on experience with real MR brain images, the intensity of white matter at the surface layer of the cerebrum is usually very close to the intensity of the gray matter in the central region of the brain, which leads to imprecise unsupervised image segmentations. Therefore, some refinement must be made using either local segmentation or a manual adjustment. In our project, we developed a specific software for the processing of such interactive image segmentation.

\section{Acknowledgment}

The work reported in this paper is a part of the project "Biochemical Markers of Traumatic Brain Injury." We were responsible for the calculation of different matters in a cerebrum. Throughout the project, I received a great deal of help for which I am greatly appreciative. I would like to thank my adviser, Dr. Yunmei Chen at the University of Florida. It is under her advisement that this project was completed. Secondly, I want to express my thanks to Dr. Ilona Schmalfuss, a Neuroradiological doctor at Shands Hospital. She spent a lot of time helping to verify the segmentation results. Without her help, it would have been impossible to finish the project. Finally, thank you to Dr. Xiaojing Ye and Dr. Feng Huang for their consultation and advice during the project. Thanks are given to all of them!

\section{Ethics Statements}

All the data used in this project are obtained from either patients (as abnormal data) or volunteers (as normal data). The use of all data is permitted for scientific research purpose only.

\section{Funding}

The project is funded by NIH/R01(\#: 7095364).

\section{References}

1. http://en.wikipedia.org/wiki/WikiGrey matter
2. http://en.wikipedia.org/wiki/WikiPeriaqueductal gray

3. Bresson X, Chan TF (2008) Non-local unsupervised varia-tional image segmentation models.

4. Houhou, etc. Semi-Supervised Segmentation based on Non-local Continuous Min-Cut. Lecture Notes in Computer Science 5567: 112-123

5. Ahmed MN, Yamany SM, Mohamed N, Farag AA, Moriarty T (2002) A modified fuzzy C-means algorithm for bias field estimation and segmentation of MRI data. IEEE Trans Med Imag 21: 193-199.

6. Chen F, Chen Y (2010) A Stochastic Variational Model for Multi-phase Sof Seg- mentation with Bias correction. Advanced Modeling and Optimization 12: 336-345.

7. Li C, Huang R, Ding Z, Gatenby C, Metaxas D (2008) A variational leve set ap- proach to segmentation and bias correction of images with intensity inhomogeneity. Miccai Lncs 5242: 1083-1091.

8. Powell S, Magnotta V, Johnson H, Vamsi K, Ronald P, et al. (2008) Registration and Machine Learning Based Automated Segmentation of Subcortical and Cerebellar Brain Structures. Neuroimage 39: 238-247.

9. Saeed N, Puri BK (2002) Cerebellum segmentation employing texture properties and knowledge based image processing: applied to normal adult controls and patients. Magnetic Resonance Imaging 20: 425-429.

10. Wang H, Chen F, Chen Y (2013) A New Multiphase Soft Segmen- tation with Adaptive Variants. Applied Computational Intelligence and Soft Computing 6.

11. Fang Li, Michael KNG, Chunming Li (2010) Variational Fuzzy Mumford-Shah Model for Image Segmentation. SIAM J Appl Math 70: 2750-2770.

12. Pham DL, Prince JL (1998) An adaptive fuzzy C-means algorithm for the image segmentation in the presence of intensity inhomogeneities. Pattern Recognit Lett 20: $57-68$.

13. Wells W, Grimson E, Kikinis R, Jolesz F (1996) Adaptive segmentation of MR data. IEEE Trans Med Imag 15: 429-442. 